\title{
Letter
}

\section{Disrupting the spatio-temporal symmetry of the electron dynamics in atmospheric pressure plasmas by voltage waveform tailoring}

\author{
Andrew R Gibson ${ }^{1,2}$ (), Zoltán Donkó ${ }^{3}{ }^{\oplus}$, Layla Alelyani ${ }^{2}$, Lena Bischoff ${ }^{1}$, \\ Gerrit Hübner $^{1}$, Jérôme Bredin ${ }^{2}$, Scott Doyle ${ }^{2}$, , Ihor Korolov $^{1}{ }^{\circledR}$, \\ Kari Niemi ${ }^{2}$ (1), Thomas Mussenbrock ${ }^{4}$, , Peter Hartmann $^{3}$,

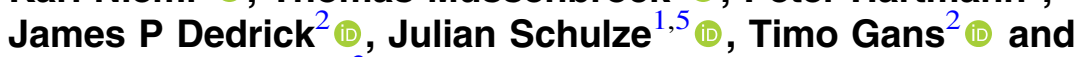 \\ Deborah O'Connell ${ }^{2}$ (1) \\ ${ }^{1}$ Institute for Electrical Engineering and Plasma Technology, Ruhr-Universität Bochum, Universitätsstraße \\ 15044801 , Bochum, Germany \\ ${ }^{2}$ York Plasma Institute, Department of Physics, University of York, York, YO10 5DD, United Kingdom \\ ${ }^{3}$ Wigner Research Centre for Physics, Hungarian Academy of Sciences, 1121 Budapest, Konkoly-Thege \\ Miklós str. 29-33, Hungary \\ ${ }^{4}$ Electrodynamics and Physical Electronics Group, Brandenburg University of Technology Cottbus- \\ Senftenberg, Cottbus, Germany \\ ${ }^{5}$ Department of Physics, West Virginia University, Morgantown, WV 26506, United States of America \\ E-mail: gibson@aept.rub.de
}

Received 24 October 2018

Accepted for publication 30 November 2018

Published 7 January 2019

\section{Abstract}

Single frequency, geometrically symmetric Radio-Frequency (RF) driven atmospheric pressure plasmas exhibit temporally and spatially symmetric patterns of electron heating, and consequently, charged particle densities and fluxes. Using a combination of phase-resolved optical emission spectroscopy and kinetic plasma simulations, we demonstrate that tailored voltage waveforms consisting of multiple RF harmonics induce targeted disruption of these symmetries. This confines the electron heating to small regions of time and space and enables the electron energy distribution function to be tailored.

Keywords: voltage waveform tailoring, atmospheric pressure plasmas, particle-in-cell simulations, phase-resolved optical emission spectroscopy, electron energy distribution functions, electron heating, radio-frequency plasmas

Non-thermal Atmospheric Pressure Plasmas (APPs) exhibit highly non-equilibrium electron dynamics, driven by complex spatio-temporal patterns of electron heating [1-4]. In geometrically symmetric systems, the sinusoidal Radio-Frequency (RF) voltages often used to drive APPs mean that these patterns are temporally and spatially symmetric. The distinct structure of the electron heating imposed by these symmetries constrains the degree to which the electron properties in APPs can be influenced by their operating conditions. This means that control of crucial parameters such as the electron energy distribution (or probability) function (EEDF/EEPF), which determines the densities and fluxes of the active species produced by APPs, is challenging.

APPs driven by multi-frequency voltage waveforms have previously been investigated using fluid simulations and experimental measurements [5-12]. These works have suggested that 
such waveforms enable a degree of favourable control of the electron properties. However, the diagnostic challenges associated with APPs mean that experiments alone cannot directly measure the EEDF, while fluid simulations do not calculate it directly, and therefore do not provide a complete description of the electron dynamics in the plasma. Kinetic plasma simulations enable the EEDF to be calculated from first principles, however, these are challenging at atmospheric pressure due to high electron-neutral collision frequencies.

Here, we demonstrate that voltage waveform tailoring i.e. the construction of specific waveform shapes by the summation of multiple RF harmonics [13-20], is capable of disrupting the temporal and spatial symmetries of electron heating in APPs. This, in turn leads to spatio-temporal confinement of the electron heating, and significantly enhanced control of the EEPF. This is studied using Particle-In-Cell simulations with Monte Carlo treatment of collision processes (PIC/MCC) and phase-resolved optical emission spectroscopy (PROES) [21, 22]. This combined approach allows for experimental validation of the simulated electron dynamics from which detailed information on the EEPF and electron heating, calculated from first principles, can be extracted. The EEPF control offered by this approach has potential for significant impact in the many technological applications of APPs in analytical [23, 24] and green chemistry [25, 26], biomedicine [27-31] and material synthesis [32, 33].

Experiments are performed using a COST Microplasma reference jet [34], with a $1 \times 1 \times 30 \mathrm{~mm}$ plasma channel, operated in $\mathrm{He}$ with $0.05 \% \mathrm{Ar}$ admixture. We use 5.0 $(99.999 \%)$ purity $\mathrm{He}$ and Ar gases with flow rates of $1 \mathrm{slm}$ and $0.5 \mathrm{sccm}$, respectively. Tailored voltage seed waveforms are generated using an arbitrary waveform generator (Keysight 33600A) and coupled to the powered electrode via a broadband amplifier (AR RF/Microwave Instrumentation 500A250A, $0.01-250 \mathrm{MHz}, 500 \mathrm{~W})$. The applied voltage is monitored by a high voltage probe (Tektronix oscilloscope probe P6015A, $75 \mathrm{MHz}$ ) connected directly to the powered electrode. The measured signal is transferred via a USB oscilloscope (Picoscope $6402 \mathrm{c} 250 \mathrm{MHz}, 5 \mathrm{Gs} \mathrm{s}^{-1}$ ) to a computer and controlled using LabVIEW software that implements an iterative feedback loop to impose the desired waveform shape at the electrode. PROES measurements are carried out using an ICCD camera (Stanford Computer Optics 4 Picos) equipped with an interference filter (750 nm FWHM of $10 \mathrm{~nm}$, Thorlabs) to observe emission from the $\operatorname{Ar}\left(2 \mathrm{p}_{1}\right)$ state at $750.4 \mathrm{~nm}$. The measurements use a gate width and step of $1 \mathrm{~ns}$. The time and space resolved electron-impact excitation rate from the ground state into the $\operatorname{Ar}\left(2 \mathrm{p}_{1}\right)$ state is calculated taking into account the natural lifetime $\tau_{\text {nat }}=22.47 \mathrm{~ns}$ [35], and collisional de-excitation by $\mathrm{He}$ and $\mathrm{Ar}$ atoms using the corresponding quenching coefficients $k_{\mathrm{He}}, k_{\mathrm{Ar}}[36]$.

Here, two classes of voltage waveform are generated by summation of consecutive harmonics using specific expressions: 'peak' and 'sawtooth-up'. Peak waveforms are attained according to [16-18]:

$$
\phi(t)=\sum_{k=1}^{N} \phi_{k} \cos \left(2 \pi k f t+\theta_{k}\right) .
$$

Here, $\phi_{k}=\phi_{\mathrm{pp}} \frac{2(N-k+1)}{(N+1)^{2}}$ and $\theta_{k}$ are the amplitudes and phase angles of the individual harmonics of a fundamental frequency $f=13.56 \mathrm{MHz}$ and $N$ is the number of harmonics. $\phi_{\mathrm{pp}}$ is the peak-to-peak voltage. For peak waveforms (see figure 1(1)) $\theta_{k}=0^{\circ}$.

To generate sawtooth-up waveforms, where the gradient of the voltage waveform is largest at the fall of the voltage (see figure 1(k)), the following expression is used [19]:

$$
\phi(t)=-\phi_{0} \sum_{k=1}^{N} \frac{1}{k} \sin (2 \pi k f t) .
$$

Here, $\phi_{0}$ is set to give the desired peak-to-peak voltage.

The simulations are based on a bounded one-dimensional in space and three-dimensional in velocity space $(1 \mathrm{~d} 3 \mathrm{v})$ electrostatic PIC/MCC code [37] that traces $\mathrm{Ar}^{+}, \mathrm{He}^{+}$and electrons. The cross-sections for electron-neutral and ionneutral collisions processes are taken from [38, 39]. For elastic $\mathrm{Ar}^{+}+\mathrm{He}$ collisions the Langevin cross-section is adopted. It is assumed that $50 \%$ of those He atoms excited by electron-impact populate metastables levels. These metastables cause Penning ionization of Ar atoms with a frequency determined by the rate coefficient for the process [40] as described in [37]. Ions created from this process are released at thermal energies, while electrons are released with an energy of $1 \mathrm{eV}$, both with isotropic initial directions in velocity space. The neutral gas temperature is set to $\mathrm{T}_{\mathrm{g}}=345 \mathrm{~K}[34,41]$. The electron reflection probability at the electrodes is set to 0.2 and the coefficients for ion-induced emission of secondary electrons from the electrodes are set for $\mathrm{Ar}^{+}$, and $\mathrm{He}^{+}$to 0.07 and 0.2 (based on lowering the value of 0.25 used in [42] according to the findings of [43, 44]), respectively. 600 equidistant grid points are used to resolve the $1 \mathrm{~mm}$ inter-electrode gap and $4 \times 10^{5}$ time steps are used within the applied voltage cycle to resolve the temporal dynamics of all plasma species.

Here, we focus on the electron heating dynamics for three driving voltage waveforms that represent the extrema of the electron heating confinement and EEPF control: sinusoidal $(N=1)$, sawtooth-up $(N=4)$ and peaks $(N=4)$. In all cases the peak-to-peak voltage is $\phi_{\mathrm{pp}}=470 \mathrm{~V}$. Figure 1 shows the measured ((a)-(c)) and computed ((d)-(f)) time and space resolved electron-impact excitation rate from the ground state to $\operatorname{Ar}\left(2 \mathrm{p}_{1}\right),((\mathrm{g})-(\mathrm{i}))$ show the computed electron heating rate and ((j)-(l)) the experimental and theoretical voltage across the plasma for each case. The powered and grounded electrodes are located at $x=0 \mathrm{~mm}$ and $x=1 \mathrm{~mm}$, respectively.

For all cases studied, the computed and measured electron-impact excitation rates are in excellent agreement. For the sinusoidal driving waveform, the excitation rate exhibits two dominant maxima of equal intensity during the 13.56 MHz cycle, indicating the spatial and temporal symmetry of the electron heating. These occur when the sheath expands at the powered electrode $(20-30 \mathrm{~ns}, 0.2-0.4 \mathrm{~mm})$ and at the grounded electrode $(60-70 \mathrm{~ns}, 0.6-0.8 \mathrm{~mm})$. Additional weaker maxima are observed on the bulk side of the collapsing sheath edge at both electrodes. Moving from 


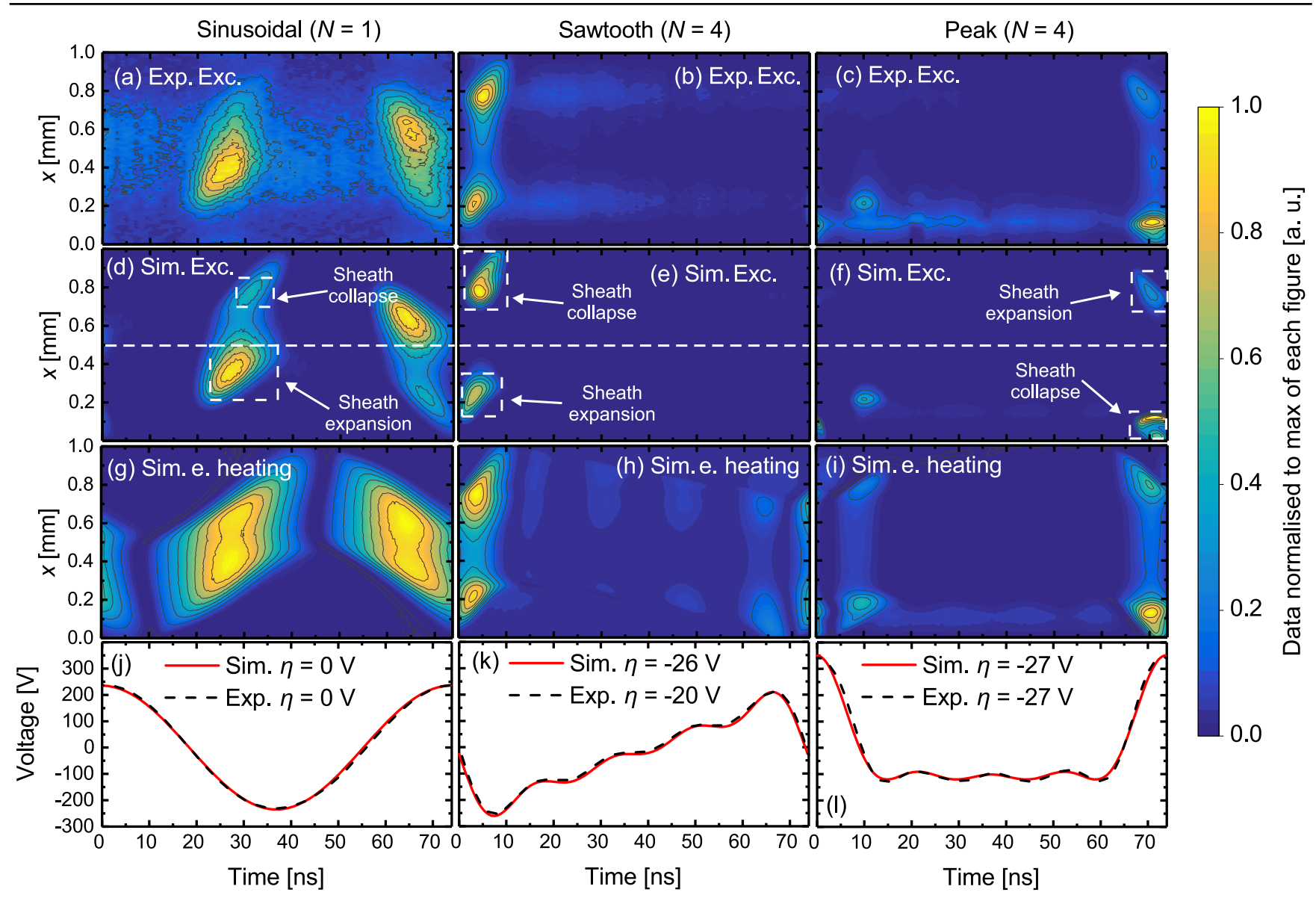

Figure 1. Time and space resolved (a)-(c) experimentally determined and (d)-(f) computed electron-impact excitation rate from the ground state to $\operatorname{Ar}\left(2 \mathrm{p}_{1}\right)$, and (g)-(i) computed electron heating rate for the voltage across the plasma shown in (j)-(l). Electron heating progresses from symmetric (sinusoidal) to temporally asymmetric (sawtooth) to spatially and temporally asymmetric (peaks). The dashed white lines and boxes in (d)-(f) represent the Regions Of Interest (ROIs) for which EEPFs are plotted in figure 3.

these maxima towards the plasma bulk, the excitation rate decreases in intensity but is still clearly apparent.

The spatio-temporal structure of the electron-impact excitation can be understood by considering the electron heating dynamics. At atmospheric pressure, electron heating is dominated by electric fields generated to overcome the collisional drag induced by frequent electron-neutral collisions [45, 46] and preserve current continuity across the plasma. For a given point in time, the current is constant in space across the plasma bulk and the electric field that forms is proportional to the loss of electron momentum due to collisions divided by the electron density [47, 48]. In general, the current across the plasma peaks shortly after the time of maximum gradient of the applied voltage waveform. Due to the relatively low electron density in the sinusoidal case, strong electric fields are generated at this time to pass the required current through the plasma. These fields are largest in regions where the electron density is smallest, i.e. close to the edges of the plasma sheath, leading to higher electron heating rates and electron-impact excitation in these regions. This mode of discharge operation is known as $\Omega$-mode [46].

For the sawtooth waveform, in both experiment and simulation, strong electron-impact excitation is observed at the start of the voltage cycle as electrons are accelerated away from the powered electrode on the 'down' phase of the waveform. Several nanoseconds later, a slightly more intense electronimpact excitation structure occurs at the grounded electrode as the adjacent sheath is collapsing. In this case, the electron heating is more localised in space to the sheath expansion and collapse regions, in contrast to the sinusoidal case where it spans over the entire plasma bulk. This is a result of the higher electron density in the sawtooth case, which reduces the electric field required to drive current through the plasma bulk. Further, the slope asymmetry of the sawtooth waveform leads to weaker electron heating when the applied voltage is increasing i.e. temporal electron heating asymmetry. Together, these factors mean that the electron energy relaxes more in the bulk plasma for the sawtooth waveform compared to the sinusoidal case. This, combined with the faster sheath collapse velocity in the sawtooth case, leads to stronger electron acceleration in the sheath collapse region to drive the required current.

The slight spatial asymmetry in electron heating leads to a small electrical asymmetry in the form of a normalised dc selfbias $\left(\bar{\eta}=\eta / \phi_{\mathrm{pp}}\right.$, where $\eta$ is the de self-bias voltage) of $\approx-4 \%$ in the experiment. The simulation predicts a normalised dc selfbias of $\approx-5 \%$, again showing excellent agreement with the measurement. This weak electrical asymmetry is in contrast to 


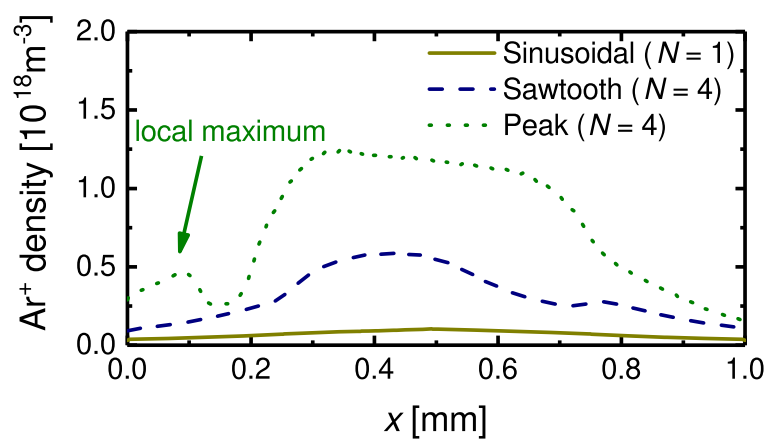

Figure 2. Time-averaged $\mathrm{Ar}^{+}$density profile for sinusoidal, sawtooth-up and peak waveforms.

electropositive plasmas driven by sawtooth waveforms at lowpressure, which typically exhibit a strong electrical asymmetry $[19,20]$. Overall in APPs, sawtooth-up waveforms are found to induce a strong temporal asymmetry (electron heating during the fall time of the voltage is greater than during the rise time) and confinement of electron heating, while remaining spatially and electrically approximately symmetric.

For peak waveforms, the electron-impact excitation exhibits both spatial and temporal asymmetry, with the electron heating confined in both space and time. Here, the major electron heating structure occurs during the sheath collapse phase from $65-74$ ns near the powered electrode $(x=0-0.2 \mathrm{~mm})$. Two weaker features are also observed, one during the sheath expansion phase several nanoseconds earlier at the grounded electrode, and the second during the sheath expansion phase at the powered electrode at the start of the voltage cycle. For the peak waveform the measured and simulated normalised dc self-bias are both $\approx-6 \%$. As with the sawtooth waveform, the absence of a strong electrical asymmetry when using a peak waveform is in contrast to lowpressure electropositive plasmas driven by peak waveforms which typically exhibit large values of $\bar{\eta}[16,17,49]$.

The stronger spatio-temporal electron heating confinement in the peak waveform case originates from the long time between the sheath expansion and collapse at the powered electrode as well as the short duration of the sheath collapse in this region. After the initial period of electron heating following sheath expansion, electrons in the bulk plasma lose energy over the majority of the voltage cycle in collisions with the background gas depleting the high-energy electron population at the discharge centre more than in the sawtooth case. As a result, when the sheath at the powered electrode collapses at the end of the voltage cycle an even stronger electric field must be generated to drive the required electron current to the powered electrode leading to pronounced electron heating in this region. Moreover, due to the short sheath collapse a large instantaneous electron current must be driven to the powered electrode to compensate the timeaveraged ion flux to this surface. This enhances the instantaneous electric field as well.

Figure 2 shows the computed time-averaged $\mathrm{Ar}^{+}$density profiles across the inter-electrode gap for the three cases. The shapes of the density profiles correlate with the spatial profiles of the electron heating i.e. the sinusoidal case exhibits a symmetric profile and the sawtooth-up case exhibits a weak asymmetry with slightly higher ion densities close to the grounded electrode. The peak case exhibits higher ion densities close to the powered electrode, with a local maximum around $x=0.1 \mathrm{~mm}$ as a result of the strong electron heating and ionization in this region. These results demonstrate that control of the spatial distribution of electron heating allows tailoring of the ion density profile, which in turn influences the flux of species to a given electrode. This leads to the opportunity for directing the ion flux towards one electrode or the other, and therefore advanced control of surface modification processes where the ion flux is an important factor. In addition, the local maximum in the ion density for the peak case influences the electron heating in this region by generating an ambipolar electric field that accelerates electrons towards the powered electrode. This acts to self-amplify the strong electron heating occurring at the sheath collapse, leading to more intense electron heating that would be present with a continuously decreasing ion density profile. Similar effects are observed in low-pressure electronegative plasmas $[45,50,51]$.

To further understand the electron energy confinement induced by each waveform, EEPFs are plotted in figure 3 for the regions of interest highlighted in figures 1(d)-(f), focusing on the excitation maxima at sheath expansion and collapse for each waveform, as well as the centre of the discharge. For the sinusoidal case, the EEPFs at sheath expansion and collapse are similar in shape. The EEPF integrated along the centre of the discharge is depleted slightly for all energies above $\approx 3 \mathrm{eV}$, exhibiting an enhanced low-energy electron population. The higher energy tails of the EEPFs at sheath expansion and collapse are a result of the strong electron heating in these regions, whereas the low-energy electron population at the centre of the gap results from regions where electrons are not actively heated.

Significant differences in the EEPFs are apparent between the asymmetric peak and sawtooth waveforms and the symmetric sinusoidal case. The strong temporal energy confinement in the sawtooth case leads to an enhancement of the high-energy tail of the EEPFs at sheath expansion and collapse and a significant reduction in the fraction of highenergy electrons present at the centre of the gap when compared to the sinusoidal case. For the peak waveform the addition of a spatial asymmetry further increases electron heating confinement. As a result, the high-energy tail of the EEPF at sheath collapse is strongly enhanced compared to that at sheath expansion and with respect to the EEPFs in the sinusoidal and sawtooth cases. The high-energy tail at the discharge centre is further depleted relative to that of the sawtooth waveform.

Figure 3(d) shows the temporally and spatially integrated EEPFs for the sinusoidal, sawtooth and peak waveforms. The EEPF in the sinusoidal case is essentially Maxwellian for energies up to $20 \mathrm{eV}$, where excitation of He begins to deplete the electron energy. When driven by peak and sawtooth waveforms the EEPFs become highly non-Maxwellian, exhibiting enhanced populations of electrons at low energies 

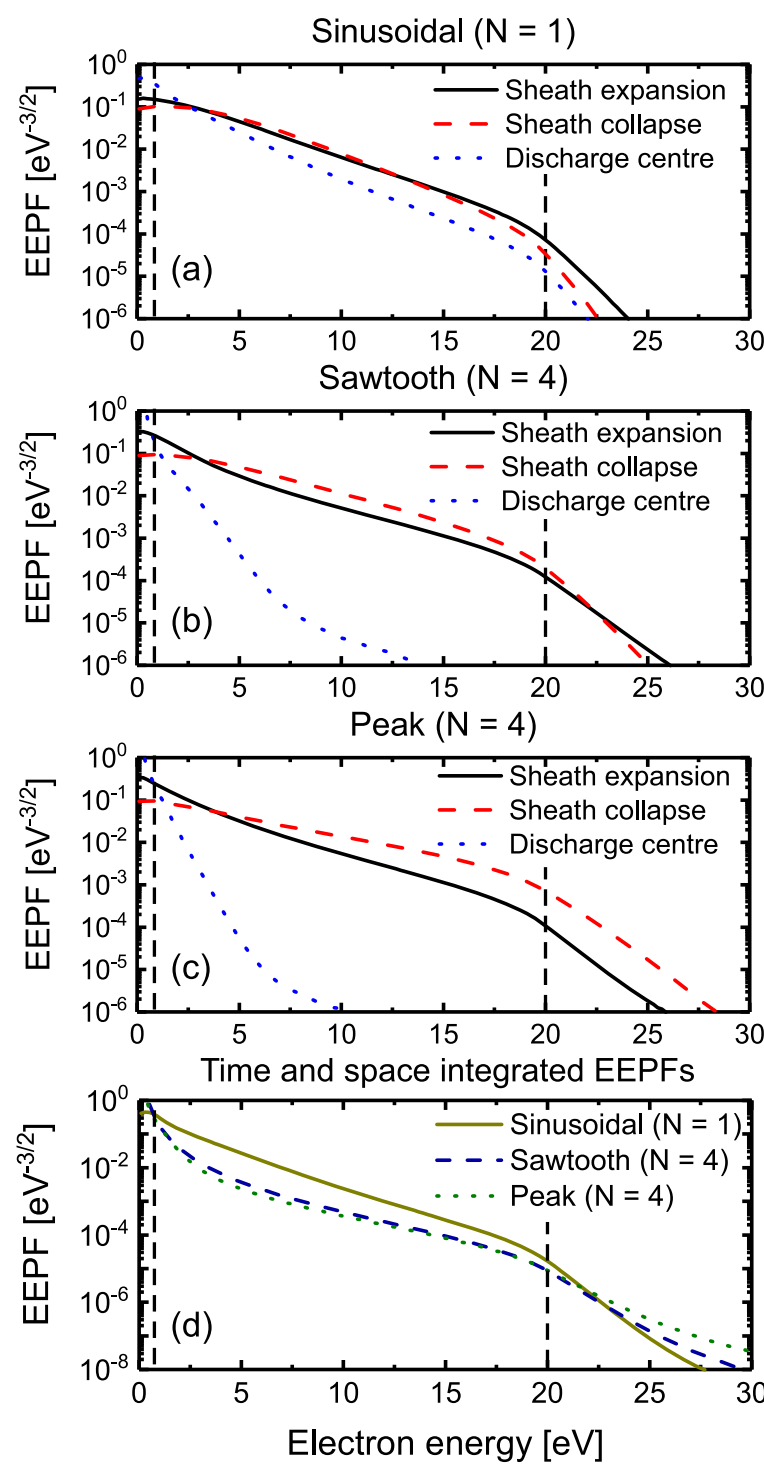

Figure 3. EEPFs for (a) sinusoidal, (b) sawtooth-up and (c) peak voltage waveforms for the ROIs shown in figure 1. (d) Time and space integrated EEPFs for the three different waveforms. The dashed vertical lines shown at 1 and $20 \mathrm{eV}$ illustrate different energy ranges discussed in the text.

and a depleted population at intermediate energies $(1-20 \mathrm{eV})$ relative to the sinusoidal case. Above $\approx 22 \mathrm{eV}$ the EEPFs for the peak and sawtooth cases exhibit a higher population of electrons compared to the sinusoidal case. The peak waveform has the highest proportion of high-energy electrons due to the strong confinement of electron heating to the sheath collapse region at the powered electrode.

In general, different energy ranges are important for different aspects of the chemistry of APPs. When small quantities of molecular gases are used, as is the case in many applications, the low-energy range $(<1 \mathrm{eV})$ is important for vibrational activation of molecules. The intermediate range $(1-20 \mathrm{eV})$ is where most direct electron-impact excitation and dissociation processes occur (for example, electron-impact dissociation of $\mathrm{O}_{2}$ requires $6-10 \mathrm{eV})$, and the high-energy range $(>20 \mathrm{eV})$ is important for formation of high-energy excited states, such as those of $\mathrm{He}$, and for direct electron-impact ionization. In this context, the EEPFs generated through breaking the symmetry of electron heating using peaks and sawtooth waveforms open up new possibilities for plasma control in applications. For example, the enhanced population of low-energy electrons for peak and sawtooth waveforms will lead to increased excitation of vibrational states, the presence of which has been associated with more efficient dissociation of molecules compared to direct electron-impact. On the other hand, the enhanced population of high-energy electrons will be useful in the production of He metastable or radiative states, which could be used as VUV light, or ionization sources.

In conclusion, we have demonstrated that tailored voltage waveforms are capable of disrupting the symmetry of electron heating in atmospheric pressure plasmas. Sawtooth driving voltage waveforms mainly induce a temporal asymmetry, confining the electron heating in time, but offer limited control of the electron heating in space. Peaks waveforms induce both spatial and temporal asymmetries, confining electron heating in both space and time. The electron heating confinement induced by these waveforms has a strong influence on the time and space resolved and time and space averaged EEPF offering the potential for enhanced control of the electron-driven chemistry in APPs, which will be crucial for future applications.

\section{Acknowledgments}

This work is supported by the Wellcome Trust [ref: 204829] through the Centre for Future Health (CFH) at the University of York, UK EPSRC grants EP/K018388/1 and EP/M508196/1, DFG grant SFB 1316 (project A4), NKFIH 119357 and 115805. The authors also acknowledge Andy Marvin and Richard Armitage for technical support.

\section{ORCID iDs}

Andrew R Gibson (ำ https://orcid.org/0000-0002-1082-4359 Zoltán Donkó (i) https://orcid.org/0000-0003-1369-6150

Scott Doyle (1) https://orcid.org/0000-0002-8741-1018

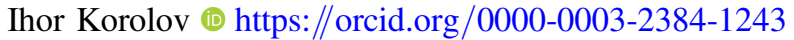

Kari Niemi i https://orcid.org/0000-0001-6134-1974

Thomas Mussenbrock (1) https://orcid.org/0000-00016445-4990

James P Dedrick @ \ttps://orcid.org/0000-0003-4353-104X Julian Schulze 나 https://orcid.org/0000-0001-7929-5734

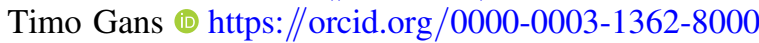

Deborah O’Connell ® ${ }^{\circ}$ https://orcid.org/0000-0002-1457-9004

\section{References}

[1] Shi J J and Kong M G 2006 Evolution of discharge structure in capacitive radio-frequency atmospheric microplasmas Phys. Rev. Lett. 96105009

[2] Iza F, Lee J K and Kong M G 2007 Electron kinetics in radiofrequency atmospheric-pressure microplasmas Phys. Rev. Lett. 9975004 
[3] Eremin D, Hemke T and Mussenbrock T 2015 Nonlocal behavior of the excitation rate in highly collisional RF discharges Plasma Sources Sci. Technol. 2444004

[4] Kawamura E, Lieberman M A and Lichtenberg A J 2016 Standing striations due to ionization instability in atmospheric pressure $\mathrm{He} / \mathrm{H}_{2} \mathrm{O}$ radio frequency capacitive discharges Plasma Sources Sci. Technol. 2554009

[5] Waskoenig $\mathbf{J}$ and Gans T 2010 Nonlinear frequency coupling in dual radio-frequency driven atmospheric pressure plasmas Appl. Phys. Lett. 96181501

[6] O'Neill C, Waskoenig J and Gans T 2012 Tailoring electron energy distribution functions through energy confinement in dual radio-frequency driven atmospheric pressure plasmas Appl. Phys. Lett. 101154107

[7] Zhou Y-J, Yuan Q-H, Li F, Wang X-M, Yin G-Q and Dong C-Z 2013 Nonequilibrium atmospheric pressure plasma jet using a combination of $50 \mathrm{kHz} / 2 \mathrm{MHz}$ dualfrequency power sources Phys. Plasmas 20113502

[8] Zhang Z L, Nie Q Y, Wang Z B, Gao X T, Kong F R, Sun Y F and Jiang B H 2016 Numerical studies of independent control of electron density and gas temperature via nonlinear coupling in dual-frequency atmospheric pressure dielectric barrier discharge plasmas Phys. Plasmas 2373501

[9] Liu D, Yang A, Wang X, Chen C, Rong M and Kong M G 2016 Electron heating and particle fluxes in dual frequency atmospheric-pressure helium capacitive discharge $J$. Phys. $D$ Appl. Phys. 49 49LT01

[10] Liu Y, Peeters F J J, Starostin S A, van de Sanden M C M and de Vries H W 2018 Improving uniformity of atmosphericpressure dielectric barrier discharges using dual frequency excitation Plasma Sources Sci. Technol. 27 01LT01

[11] Park S, Choe W, Moon S Y and Shi J J 2018 Electron information in single- and dual-frequency capacitive discharges at atmospheric pressure Sci. Rep. 87516

[12] Zhang Z L, Nie Q Y, Zhang X N, Wang Z B, Kong F R, Jiang B H and Lim J W M 2018 Ionization asymmetry effects on the properties modulation of atmospheric pressure dielectric barrier discharge sustained by tailored voltage waveforms Phys. Plasmas 25043502

[13] Lafleur T 2015 Tailored-waveform excitation of capacitively coupled plasmas and the electrical asymmetry effect Plasma Sources Sci. Technol. 2513001

[14] Heil B G, Czarnetzki U, Brinkmann R P and Mussenbrock T 2008 On the possibility of making a geometrically symmetric RF-CCP discharge electrically asymmetric J. Phys. D Appl. Phys. 41165202

[15] Zhang Y, Zafar A, Coumou D J, Shannon S C and Kushner M J 2015 Control of ion energy distributions using phase shifting in multi-frequency capacitively coupled plasmas J. Appl. Phys. 117233302

[16] Schulze J, Schüngel E, Donkó Z and Czarnetzki U 2011 The electrical asymmetry effect in multi-frequency capacitively coupled radio frequency discharges Plasma Sources Sci. Technol. 2015017

[17] Berger B, Brandt S, Franek J, Schüngel E, Koepke M, Mussenbrock T and Schulze J 2015 Experimental investigations of electron heating dynamics and ion energy distributions in capacitive discharges driven by customized voltage waveforms J. Appl. Phys. 118223302

[18] Derzsi A, Bruneau B, Gibson A R, Johnson E, O'Connell D, Gans T, Booth J-P and Donkó Z 2017 Power coupling mode transitions induced by tailored voltage waveforms in capacitive oxygen discharges Plasma Sources Sci. Technol. 2634002

[19] Bruneau B, Gans T, O'Connell D, Greb A, Johnson E V and Booth J P 2015 Strong ionization asymmetry in a geometrically symmetric radio frequency capacitively coupled plasma induced by sawtooth voltage waveforms Phys. Rev. Lett. 114125002

[20] Bruneau B et al 2015 Effect of gas properties on the dynamics of the electrical slope asymmetry effect in capacitive plasmas: comparison of $\mathrm{Ar}, \mathrm{H}_{2}$ and $\mathrm{CF}_{4}$ Plasma Sources Sci. Technol. 25 01LT02

[21] Schulze J, Schüngel E, Donkó Z, Luggenhölscher D and Czarnetzki U 2010 Phase resolved optical emission spectroscopy: a non-intrusive diagnostic to study electron dynamics in capacitive radio frequency discharges $J$. Phys. D Appl. Phys. 43124016

[22] Gans T, O'Connell D, der Gathen V and Waskoenig J 2010 The challenge of revealing and tailoring the dynamics of radiofrequency plasmas Plasma Sources Sci. Technol. 1934010

[23] Monge M E, Harris G A, Dwivedi P and Fernandez F M 2013 Mass spectrometry: recent advances in direct open air surface sampling/ionization Chem. Rev. 113 2269-308

[24] Rumbach P, Bartels D M, Sankaran R M and Go D B 2015 The solvation of electrons by an atmospheric-pressure plasma Nat. Commun. 67248

[25] Bogaerts A and Neyts E C 2018 Plasma technology: an emerging technology for energy storage ACS Energy Lett. 3 1013-27

[26] Mehta P, Barboun P, Herrera F A, Kim J, Rumbach P, Go D B, Hicks J C and Schneider W F 2018 Overcoming ammonia synthesis scaling relations with plasma-enabled catalysis Nat. Catal. 1 269-75

[27] Kong M G, Kroesen G, Morfill G, Nosenko T, Shimizu T, van Dijk J and Zimmermann J L 2009 Plasma medicine: an introductory review New J. Phys. 11115012

[28] von Woedtke T, Reuter S, Masur K and Weltmann K-D 2013 Plasmas for medicine Phys. Rep. 530 291-320

[29] Hirst A M, Simms M S, Mann V M, Maitland N J, O'Connell D and Frame F M 2015 Low-temperature plasma treatment induces DNA damage leading to necrotic cell death in primary prostate epithelial cells Br J Cancer 112 1536-45

[30] Gorbanev Y, O'Connell D and Chechik V 2016 Non-thermal plasma in contact with water: the origin of species Chem. Eur. J. 22 3496-505

[31] Lu X, Naidis G V, Laroussi M, Reuter S, Graves D B and Ostrikov K 2016 Reactive species in non-equilibrium atmospheric-pressure plasmas: Generation, transport, and biological effects Phys. Rep. 630 1-84

[32] Kortshagen U R, Sankaran R M, Pereira R N, Girshick S L, Wu J J and Aydil E S 2016 nonthermal plasma synthesis of nanocrystals: fundamental principles, materials, and applications Chem. Rev. 116 11061-127

[33] Maguire P, Rutherford D, Macias-Montero M, Mahony C, Kelsey C, Tweedie M, Pérez-Martin F, McQuaid H, Diver D and Mariotti D 2017 Continuous In-flight synthesis for on-demand delivery of ligand-free colloidal gold nanoparticles Nano Lett. 17 1336-43

[34] Golda J et al 2016 Concepts and characteristics of the COST Reference Microplasma Jet J. Phys. D Appl. Phys. 4984003

[35] Kramida A, Ralchenko Y, Reader J and (the NIST ASD Team) 2018 NIST Atomic Spectra Database (ver. 5.5.6), https:// physics.nist.gov/asd (25 July 2018). National Institute of Standards and Technology, Gaithersburg,MD (https://doi. org / 10.18434/T4W30F)

[36] Sadeghi N, Setser D W, Francis A, Czarnetzki U and Döbele H F 2001 Quenching rate constants for reactions of $\operatorname{Ar}(4 \mathrm{p}[1 / 2] 0,4 \mathrm{p}[1 / 2] 0,4 \mathrm{p}[3 / 2] 2$, and $4 \mathrm{p}[5 / 2] 2)$ atoms with 22 reagent gases J. Chem. Phys. $1153144-54$

[37] Donkó Z, Hamaguchi S and Gans T 2018 The effect of photoemission on nanosecond helium microdischarges at atmospheric pressure Plasma Sources Sci. Technol. 27 54001 
[38] Biagi S F 2004 Cross section Compilation, version 7.1 http:// lxcat.net

[39] Phelps A V 1994 The application of scattering cross sections to ion flux models in discharge sheaths J. Appl. Phys. 76 747-53

[40] Pouvesle J M, Khacef A, Stevefelt J, Jahani H, Gylys V T and Collins C B 1988 Study of two-body and three-body channels for the reaction of metastable helium atoms with selected atomic and molecular species J. Chem. Phys. 88 3061-71

[41] Wijaikhum A, Schröder D, Schröter S, Gibson A R, Niemi K, Friderich J, Greb A, Schulz-von der Gathen V, O'Connell D and Gans T 2017 Absolute ozone densities in a radio-frequency driven atmospheric pressure plasma using two-beam UV-LED absorption spectroscopy and numerical simulations Plasma Sources Sci. Technol. 26115004

[42] Sakiyama Y and Graves D B 2006 Corona-glow transition in the atmospheric pressure RF-excited plasma needle J. Phys. D Appl. Phys. 393644

[43] Donkó Z, Hartmann P and Kutasi K 2006 On the reliability of low-pressure de glow discharge modelling Plasma Sources Sci. Technol. 15 178-86

[44] Kutasi K, Hartmann P, Bánó G and Donkó Z $2005 \mathrm{He}_{2}^{+}$ molecular ions in helium glow discharges: the effect of bulk electron temperature Plasma Sources Sci. Technol. 14 S1-8

[45] Schulze J, Derzsi A, Dittmann K, Hemke T, Meichsner J and Donkó Z 2011 Ionization by drift and ambipolar electric fields in electronegative capacitive radio frequency plasmas Phys. Rev. Lett. 107275001

[46] Hemke T, Eremin D, Mussenbrock T, Derzsi A, Donkó Z, Dittmann K, Meichsner J and Schulze J 2013 Ionization by bulk heating of electrons in capacitive radio frequency atmospheric pressure microplasmas Plasma Sources Sci. Technol. 2215012

[47] Schulze J, Donkó Z, Lafleur T, Wilczek S and Brinkmann R P 2018 Spatio-temporal analysis of the electron power absorption in electropositive capacitive RF plasmas based on moments of the Boltzmann equation Plasma Sources Sci. Technol. 2755010

[48] Lafleur T, Chabert P and Booth J P 2014 Electron heating in capacitively coupled plasmas revisited Plasma Sources Sci. Technol. 2335010

[49] Derzsi A, Korolov I, Schüngel E, Donkó Z and Schulze J 2013 Electron heating and control of ion properties in capacitive discharges driven by customized voltage waveforms Plasma Sources Sci. Technol. 2265009

[50] Liu Y-X, Schüngel E, Korolov I, Donkó Z, Wang Y-N and Schulze J 2016 Experimental observation and computational analysis of striations in electronegative capacitively coupled radio-frequency plasmas Phys. Rev. Lett. 116255002

[51] Brandt $\mathrm{S}$ et al 2016 Electron power absorption dynamics in capacitive radio frequency discharges driven by tailored voltage waveforms in $\mathrm{CF}_{4}$ Plasma Sources Sci. Technol. 25 45015 